\title{
Physiological and Psychological Changes at the End of the Soccer Season in Elite Female Athletes
}

\author{
by \\ Jose Morales ${ }^{1}$, Vicenç Roman², Alexandre Yáñez ${ }^{1}$, Mònica Solana-Tramunt ${ }^{1}$, \\ Juan Álamo ${ }^{3}$, Antón Fíguls ${ }^{2}$
}

\begin{abstract}
This study compares and describes relationships among stress-recovery indices, the heart rate variability index, and the Cooper and Yo-Yo IR1 tests among female soccer players during the last six weeks of the competitive season. Sixteen female soccer players engaged in a pre-test of all of the variables. After having their training monitored for six weeks, a post-test was administered. The results revealed significant $(p<0.05)$ differences in the specific stress-recovery scales of the RESTQ-sport and in the frequency-domain variables of the $H R V$, although there were no significant differences in the general stress or general recovery scales. The Yo-Yo IR1 test, the Cooper test scores, and the means of the time-domain HRV variables did not exhibit any significant differences between the pre-and the post-test. The RMSSD variations exhibited very large and large correlations with the performance test and the RESTQ-sport variables, respectively. The variations in the HRV frequency-domain variables exhibited significant moderate and large correlations among the variations of the RESTQ-sport scales. Monitoring athletes at the end of the season may reveal contradictions between some variables. To help with the interpretation of these scales, some external aspects, such as athlete strain and monotony of training, should be considered.
\end{abstract}

Key words: RESTQ-sport, HRV, Yo-Yo IR1, Cooper test, monotony.

\section{Introduction}

The competitive season in soccer is characterized by different performance states of players due to the application of different training loads (TLs) that affect the stress-recovery (SR) status of each athlete (Dupont et al., 2004; Flatt et al., 2017). According to Thorpe et al. (2015), TL patterns vary considerably between the playing season and the preseason because of differences in training and match volume. However, to the best of our knowledge the number of studies involving monitoring TLs and SR status of female soccer players using various tools simultaneously inseason is much smaller than the number of studies focusing on male soccer players, and smaller than those concerning athletes participating in other sports. It is accepted that the final period of the season is decisive for evaluating the final success of a soccer team. In this sense, the control of TLs and their psycho-physiological effects during this period has an impact on players' performance, and knowledge of these data provides valuable information to optimize the training process (Borresen and Lambert, 2009).

Studies of team sports, especially soccer, have been characterized by attempts to quantify training and competition loads using different systems. Nowadays, there are different options for TL assessment during a soccer match, including the GPS which allows researchers to assess several TL indices (Buchheit et al., 2014; Malone et al., 2015), heart rate (HR) monitors, blood lactate or gas exchange measurements (Borresen and Lambert,

\footnotetext{
1 - Faculty of Psychology, Education Sciences and Sport Blanquerna-Ramon Llull University, Barcelona, Spain.

2 - Institut Francesc Macià. Departament d'ensenyament. Generalitat de Catalunya. Spain.

3 - Department of physiology, University of Barcelona, Barcelona, Spain.
} 
2009), and other inexpensive and easy-to-apply options such as the Rate of Perceived Exertion (RPE) Session (RPEsession) (Foster et al., 1995) that have been used in previous studies focusing on soccer players (Algrøy et al., 2011).

Heart rate variability (HRV) measurements have been used to measure internal TLs and overtraining in soccer (Flatt et al., 2017). Heart rate variability refers to variations in beat intervals or correspondingly in the instantaneous HR in different situations. Various studies have used HRV to better understand the status of the Autonomic Nervous System (ANS) and stress induced by TLs; this stress interferes with the sympathetic-parasympathetic ANS balance. The relationship between ANS and HRV differs during activity and immediate recovery situations compared with during recovery periods (Kaikkonen et al., 2007). Accurate HRV assessments require stationary conditions; therefore, it is preferable to measure HRV a significant amount of time before or after exercise or during immediate recovery states (Kaikkonen et al., 2007; Task Force of the European Society of Cardiology and the North American Society of Pacing and Electrophysiology, 1996).

From a psychological perspective, different instruments are useful for assessing stress-recovery situations and internal TLs during training and these instruments can be also used to detect a possible overtraining situation or to prevent an injury. Kellmann and Kallus (2001) developed the Recovery Stress Questionnaire for Athletes (RESTQ-sport), an instrument to measure SR balance in athletes. The RESTQ-sport has been used in soccer (Laux et al., 2015), and its application is important for both competitions and training sessions because it enables evaluations of the current situation and expectations of performance in upcoming games or training sessions.

Since performance in soccer depends on many factors, the use of a variety of psychological and physiological variables, together with a detailed description of the characteristics of training, is justified. Therefore, the objectives of this study were: (i) to describe the TL of the last mesocycle of the season, (ii) to compare the results of performance, psychological and physiological tests before and after the final mesocycle, and (iii) to establish a relationship between the chosen variables of the different domains evaluated. We hypothesized that there would be significant differences between the initial and the final values due to the accumulated effect of training.

\section{Methods}

\section{Participants}

Sixteen professional female soccer players from the Club de Fútbol Levante-Las Planas (Barcelona) competing in the Spanish First Division (age: $23.25 \pm 5.07$ years; body mass: 60.94 $\pm 9.55 \mathrm{~kg}$, body height: $1.61 \pm 6.89 \mathrm{~m}$, and body mass index: $23.55 \pm 2.11 \mathrm{~kg} / \mathrm{m}^{2}$ ) participated in the study.

After being fully informed verbally and in writing of the purposes and potential risks of the study, the subjects provided their written consent to participate in the investigation. The study and its protocol were reviewed and approved by the Ramon Llull University internal review board and conducted in accordance with the latest version of the Declaration of Helsinki.

\section{Design and Procedures}

We collected data during pre-test and posttest sessions, just after Easter Week holidays. The data collection occurred one week immediately after the second mesocycle of the season. Between tests, the soccer players continued with their last mesocycle training program, and the re-test was performed before the last week of the competitive period.

All testing sessions were completed 2 hours prior to the start of training. During the testing sessions, we measured HRV and collected RESTQsport questionnaire information. The subjects also performed the Cooper test and, after 48 hours, the Yo-Yo IR1 test. The researchers responsible for data collection were blinded to the participants and the information was codified and statistically treated by other researchers.

Six players were excluded to avoid HRV disturbances because they were in their luteal menstrual period; this phase is known to affect HRV results (de Zambotti et al., 2013).

HRV analysis

In order to obtain accurate data, we applied The Task Force of the European Society of Cardiology and the North American Society of Pacing and Electrophysiology criteria to all of the HRV measurements. The HRV was always recorded at $5 \mathrm{pm}$ ( 2 hours before training), and the 
athletes were instructed to avoid any stimulants, thermogenics and alcohol for at least 48 hours. The testing room conditions were standardized (quiet, no distractions, temperature of $20-22^{\circ} \mathrm{C}$ ).

The subjects were asked to remain still and breathe at a pace between 10-12 cycles per minute without speaking or making any movements. These conditions were maintained during the preand the post-test. The female athletes were fitted with HR monitors and given 5 minutes to rest in a supine position prior to the data collection. Interbeat (RR) interval recordings were obtained during the next 5 minutes of each subject using a validated portable HR monitor (Polar ${ }^{\circledR}$ RS810, Kempele, Finland). This series of HR monitors had been validated for HRV recordings (Giles et al., 2016). The RR recordings were downloaded using the accompanying Polar software (Polar ${ }^{\circledR}$ Precision Performance) and exported for later analysis of time- and frequency-domain measures of HRV using Kubios v2.0 HRV software (Biosignal Analysis and Medical Imaging Group at the Department of Applied Physics, University of Kuopio, Kuopio, Finland).

Based on the original RR intervals, the following variables were calculated: the standard deviation of the RR-intervals (SDNN), the rootmean-square successive difference of intervals (RMSSD).

We analyzed different HRV variables in the frequency domain, which were obtained by analyzing the spectral analysis density of the RR signal. As recommended by the Task Force (Task Force of the European Society of Cardiology and the North American Society of Pacing and Electrophysiology 1996), we performed spectral analysis with a Fast Fourier Transformer to quantify the power spectral density of the lowfrequency (LF; 0.04-0.15 Hz) and high-frequency (HF; 0.16-0.40 Hz) bands.

The HF band reflects vagal modulation, and the LF band reflects both sympathetic and parasympathetic influences. Furthermore, the $\mathrm{LF} / \mathrm{HF}$ ratio was examined as an indicator of sympatho-vagal balance (Bosquet et al., 2008).

RESTQ-sport test analysis

The RESTQ-sport recovery-stress questionnaire was used (Kellmann and Kallus, 2001) to determine the perceived stress of seasonal activities.

The soccer players had to answer 77 questions related to 19 different scales. In addition, the RESTQ-sport scores were classified as general stress scores (mean of 7 scales), general recovery scores (mean of 5 scales), sport-specific stress scores (mean of 3 scales), and sport-specific recovery scores (mean of 4 scales). The final score of each scale was the mean of the points obtained in each item. Each subject's answer was checked based on a Likert scale with values ranging from 0 6.

The Spanish version of the RESTQ-sport was used, which had been validated by Gonzalez-Boto et al. (2009). This version demonstrated high reliability with a Cronbach Alpha index ranging from 0.77 to 0.94 and significant correlations with the Profile of Mood States questionnaire and the RESTQ-sport scales.

Performance tests

The Cooper test was conducted taking into account the aspects described by McArdle et al. (2010) (i.e., a 200-m track with markings every 50 $\mathrm{m})$. All the subjects were familiarized with the test protocol, and they performed a warm up of $2 \mathrm{~min}$ of running and $3 \mathrm{~min}$ of stretching. To begin the test, a "GO" command was given and the timer was started. During the test, athletes were kept informed of the remaining time at the end of each lap $(200 \mathrm{~m})$. Once the test ended after 12 minutes, the distance that the athlete covered, rounded to the nearest $50 \mathrm{~m}$ mark, was registered.

The Yo-Yo IR1 test was performed following the protocol proposed by Bangsbo et al. (2008) after completion of a specific warm-up. The Yo-Yo IR1 test consisted of repeated 20-m runs back and forth between two markers with a progressive increase in speed, which was governed by an audio player. Between each $40-\mathrm{m}$ run, the athlete recovered with $10 \mathrm{~s}$ of jogging (shuttle runs of $2 \times 5 \mathrm{~m}$ ). The test consisted of 4 runs at $10-13 \mathrm{~km} \cdot \mathrm{h}^{-1}(0-160 \mathrm{~m})$ and another 7 runs at $13.5-14.0 \mathrm{~km} \cdot \mathrm{h}^{-1}(160-440 \mathrm{~m})$. From this point on, the players completed 8 additional runs, each $0.5 \mathrm{~km} \cdot \mathrm{h}^{-1}$ faster than the previous one. The test was completed when the athlete reached voluntary exhaustion or failed to maintain her running pace in synchrony with the audio recording (i.e., she failed to achieve the markers twice in the same stage). The Yo-Yo IR1 course lines were marked with cones $20 \mathrm{~m}$ apart with additional cones set $5 \mathrm{~m}$ behind the starting line for use during recovery.

The athletes wore HR monitors to record the 
final HR attained during the test.

Training program characteristics

The athletes underwent the same training program for 6 weeks and focused on specific objectives of the end of the competitive period. The TL was characterized by using specific highintensity and decreased volume loads. The recovery time was sufficient to ensure that the athletes were fit during competition (Figure 1).

We monitored internal TLs using the HRbased approach of Edwards (1993) and the RPEbased approach of Foster et al. (2001).

The method of Edwards (1993) is based on the time accumulated in five HR zones and is calculated analyzing each zone (zone 1: $50-60 \%$ of $\mathrm{HR}_{\max }$; zone 2: $60-70 \%$ of $\mathrm{HR}_{\max }$; zone 3: $70-80 \%$ of HRmax; zone 4: 80-90\% of HR $\max$; zone $5: 90-100 \%$ of $H_{R}$ max). The calculation was made by summing the number of minutes in each zone multiplied by the zone number. The HR assessment was made using a portable HR monitor (Polar ${ }^{\circledR}$ RS810), and the recordings were downloaded using the accompanying Polar $^{\circledR}$ software.

The method proposed by Foster et al. ( 2001) requires subjects to rate the intensity of training 30 min after the end of the session using the Borg's Category Ratio-10 (CR-10) RPE scale. The TL is calculated by multiplying the duration of exercise by the RPEsession score.

We collected RPEsession scores and the scores of the Edwards method and calculated the mean of the week-long values of all of the subjects. At the same time, the week-long monotony was calculated according to Foster (1998) by dividing the mean of the week-long TL from RPEsession by its standard deviation. The monotony is a measurement of variability of training across one week. If the TL is always high or always low during the week, the monotony value will be high. However, if high and low TLs are intercalated during the training week, the monotony score will be moderate or low. The training strain is a value that represents the stress to which the athlete was exposed during the entire training week. It is obtained by multiplying the weekly TL (including the load of the competition) by the training monotony score. A week of higher TLs along with a high monotony score yields very large training effort values.

\section{Statistical analysis}

We performed statistical analyses using the
Statistical Package for Social Science version 22.0 (SPSS, Inc., Chicago, IL, USA). A significance level of $p<0.05$ was used for all the tests. The ShapiroWilk test was applied to test for a normal distribution of the data. A repeated-measures ANOVA of internal TL variables was carried out to check for differences during the six weeks of training. We relied on a paired t-test to compare the differences between the pre- and post-test scores for all dependent variables (HRV, RESTQ-sport, and the performance test). Moreover, Pearson product-moment correlations were used to quantify the relationships between pre- and posttest changes in HRV, RESTQ-sport and performance variables. Finally, we evaluated the correlation of all the dependent variables in the post-test with the final result of the monotony and the training strain of the last week of training.

\section{Results}

\section{Training load variables}

A main effect of the time factor was observed. Significant differences in the variables quantifying the TLs according to the mesocycle week and the Edwards method $\left(\mathrm{F}_{(5,45)}=39.45, p<0.05 ; \eta^{2} \mathrm{p}=0.39\right)$ and RPEsession $\left(\mathrm{F}_{(5,45)}=47.36, p<0.05 ; \eta^{2} \mathrm{p}=0.31\right)$ were noted. Figure 1 shows the values of the different training variables. For the variables that did not fulfill sphericity based on the Mauchly test, the degrees of freedom were adjusted using the Huynh-Feldt method. The monotony $\left(\mathrm{F}_{(3.55,32.88)}=\right.$ 6.67, $\left.p<0.05 ; \eta^{2} \mathrm{p}=0.29\right)$ and the training strain $\left(\mathrm{F}_{(3.15,30.18)}=16.65, p<0.05 ; \eta^{2} \mathrm{p}=0.21\right)$ also revealed significant differences between the different weeks of training. Pairwise comparisons were performed using the Bonferroni post hoc tests and are shown in Figure 1.

HRV variables

The means of the HRV variables did not exhibit any significant differences in either the time domain or in the pre- or post-test. We found significant differences only in the frequency analyses that used normalized units. There was a significant increase $(p<0.05)$ in LF $\left(\mathrm{t}_{15}=4.19 ; p=\right.$ $0.001 ; \mathrm{r}=0.73)$ and the LF/HF ratio $\left(\mathrm{t}_{15}=4.4 ; p=\right.$ $0.001 ; \mathrm{r}=0.75)$ and a significant decrease in $\mathrm{HF}\left(\mathrm{t}_{15}\right.$ $=4.23 ; p=0.001 ; \mathrm{r}=0.74$ ) between the pre- and the post-test. Descriptive analyses are provided in Table 1.

\section{Stress/recovery variables}

Contrast analysis was used to compare the 
means of RESTQ-sport results and the stressrecovery perception of the subjects. There were significant differences in sport-specific stress $\left(\mathrm{t}_{15}=\right.$ $4.95 ; p=0.001 ; \mathrm{r}=0.79$ ) and sport-specific recovery $\left(\mathrm{t}_{15}=-5.9 ; p=0.001 ; \mathrm{r}=0.84\right)$ between the pre- and the post-test scores. No significant differences were observed in the general stress or the general recovery scales (Figure 2).

Performance variables

There were no significant differences between the means of the performance tests in the pre- and the post-test scores. Both tests' scores had increased, yet not significant results in the Cooper test $\left(\mathrm{t}_{15}=-2.08 ; p=0.58\right)$ and the Yo-Yo IR1 test $\left(\mathrm{t}_{15}=\right.$ $-1.73 ; p=0.11$ ) (Figure 3).

\section{Correlations between dependent variables}

A very large, significant correlation was found between $\triangle$ RMSSD and $\triangle \mathrm{Yo}$-YoIR1 $(\mathrm{r}=0.84$; $p=0.02)$ and the Cooper test $(r=0.78 ; p=0.03)$. $\triangle$ RMSSD also exhibited a large and significant correlation with the RESTQ-sport variables, $\Delta$ general stress $(-0.61 ; p=0.01), \Delta$ specific stress $(\mathrm{r}=$ $0.58 ; p=0.01), \Delta$ general recovery $(\mathrm{r}=0.64 ; p=0.003)$, and $\Delta$ specific recovery $(\mathrm{r}=0.50 ; p=0.009)$. The other HRV time-domain variables exhibited nonsignificant, but moderate correlations with the RESTQ-sport and the performance test variables $(\mathrm{r}=0.34-0.45 ; p>0.05)$.

The variations in the HRV frequency-domain variables exhibited some significant moderate and large correlations among the variations of RESTQsport, $\Delta \mathrm{HF}$ and $\Delta$ specific recovery $(\mathrm{r}=0.68 ; p=$ $0.007), \Delta \mathrm{HF}$ and $\Delta$ specific stress $(\mathrm{r}=0.61 ; p=0.02)$, $\Delta \mathrm{HF}$ and $\Delta$ general stress $(\mathrm{r}=0.55 ; p=0.02), \Delta \mathrm{LF} / \mathrm{HF}$ and $\Delta$ specific recovery $(\mathrm{r}=0.48 ; p=0.01), \Delta \mathrm{LF} / \mathrm{HF}$ and $\Delta$ specific stress $(\mathrm{r}=0.31 ; p=0.04), \Delta \mathrm{LF} / \mathrm{HF}$ and $\Delta$ general stress $(\mathrm{r}=0.55 ; p=0.04), \Delta \mathrm{LF}$ and $\Delta$ specific stress $(r=0.38 ; p=0.04)$. The remainder of the variables did not exhibit any significant correlations; all were small or moderate $(\mathrm{r}=0.16-$ $0.36 ; p>0.05$ ).

Training monotony exhibited high and significant correlations with some variables of RESTQ-sport, specific stress $(\mathrm{r}=0.71 ; p=0.02)$ and specific recovery $(\mathrm{r}=-0.66 ; p=0.03)$. There were moderate correlations with the variable HRV of the frequency domain HF $(r=0.48), \mathrm{LF}(\mathrm{r}=0.41)$ and $\mathrm{LF} / \mathrm{HF}(\mathrm{r}=0.45)$. The remaining HRV variables did not exhibit any significant correlations and were low or very low in value.

Training strain exhibited high and moderate correlations with RMSSD ( $\mathrm{r}=-0.70 ; \mathrm{p}=0.01)$, Yo-Yo IR1 $(r=0.44)$, and the Cooper test $(r=0.41)$. The remaining variables did not exhibit any significant correlations and were low or very low in value.

Table 1

Heart rate and HRV variable comparisons between the pre- and post-test.

\begin{tabular}{cll}
\hline Variable & Pre-test & Post-test \\
\hline Time-domain HRV & $\mathbf{5 8 . 2 5 ( 2 . 4 2 )}$ & $\mathbf{5 8 . 1 1 ( 3 . 1 2 )}$ \\
MeanRR (ms) & & \\
STDRR (ms) & $957.15(42.94)$ & $917.23(64.04)$ \\
MeanHR (1/min) & $65.96(9.07)$ & $96.03(11.68)$ \\
STDHR (1/min) & $62.84(2.46)$ & $76.97(11.08)$ \\
RMSSD (ms) & $4.11(0.35)$ & $10.88(2.4)$ \\
Frequency-domain HRV & $72.56(14.36)$ & $76.6(9.11)$ \\
LF $($ u.n. $)$ & & $60.84(3.63)^{*}$ \\
HF(u.n.) & $43.12(3.77)$ & $38.47(3.63)^{*}$ \\
LF/HF ratio & $56.14(3.72)$ & $1.58(0.22) *$
\end{tabular}

The data are expressed as mean (standard error of the mean).

*Indicates a significant difference $(p<0.05)$ between the pre-and post-test. 


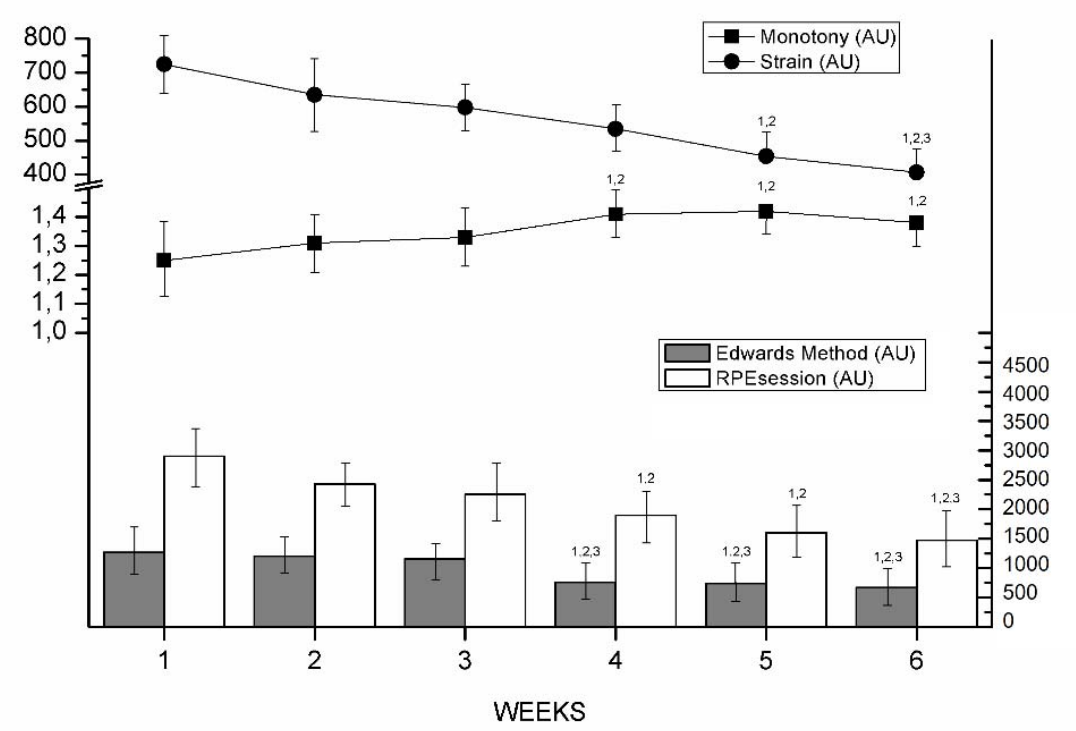

Figure 1

Quantification of TLs of the team's weekly mean. ${ }^{1}$ Means significant differences compared to week 1, ${ }^{2}$ Means significant differences compared to week 2, and ${ }^{3}$ Means significant differences compared to week 3 .

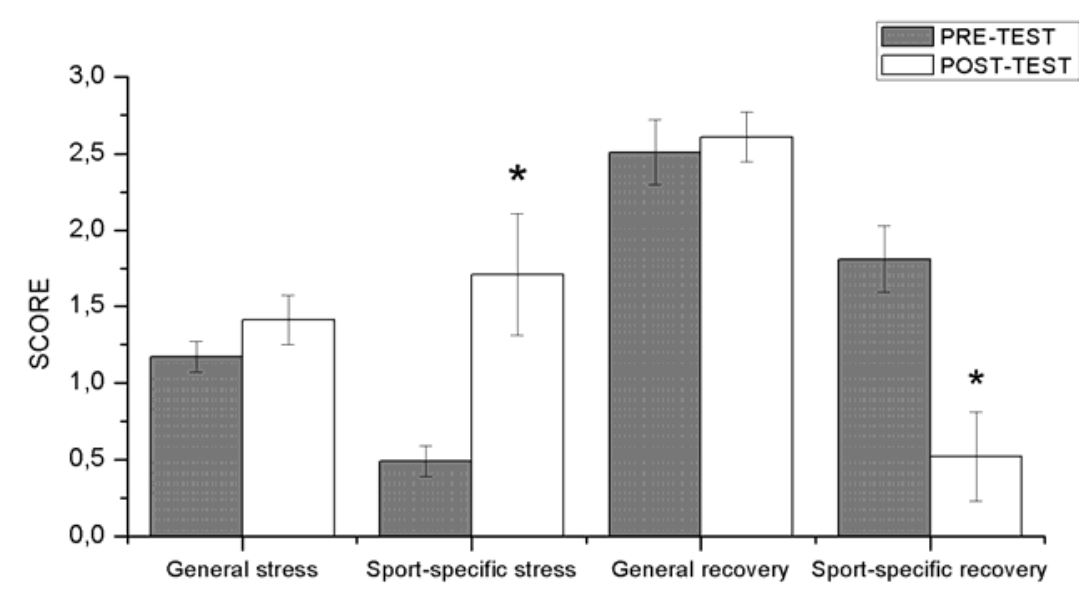

Figure 2

Differences in RESTQ-sport scales (general stress scores, general recovery, sport-specific stress, and sport-specific recovery). The columns represent the mean and the error bars represent the standard error of the mean. *Indicates significant differences $(p<0.05)$. 


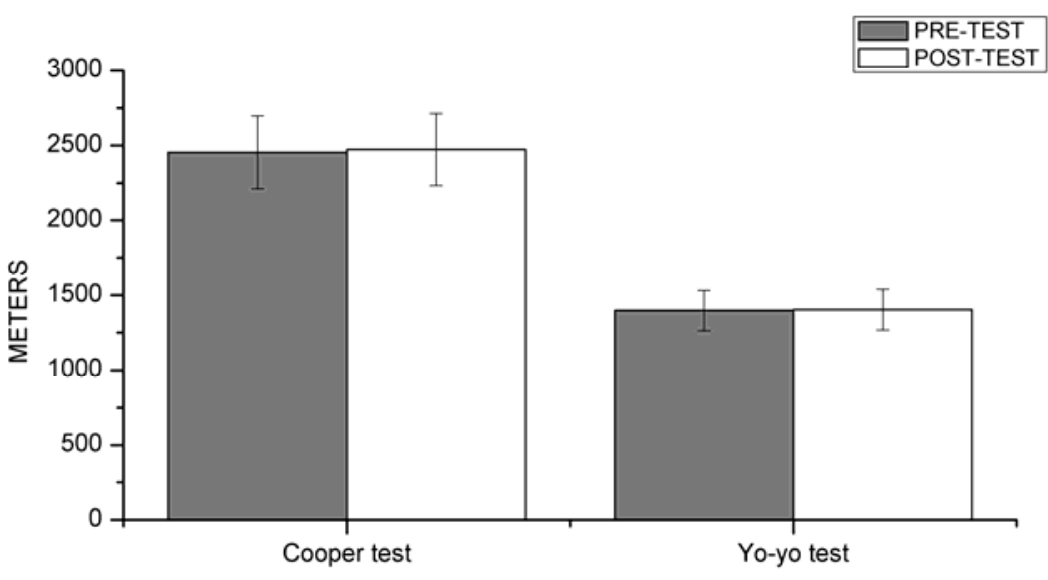

Figure 3

Differences in performance tests (Cooper and Yo-Yo IR1). The columns represent the mean and the error bars represent the standard error of the mean.

${ }^{*}$ Indicates significant differences $(p<0.05)$.

\section{Discussion}

The results revealed significant changes between the initial measurement and some of the analyzed variables at the end of the female soccer players' season. These changes can be observed in the differences between the RESQT-sport scores and the HRV results at the beginning and the end of the analyzed mesocycle. In contrast, there were no differences between the pre-test and the posttest in the Cooper test or the Yo-Yo IR1 results, although the planning strategy was focused on tapering at the end of the season to obtain peak performance in the athletes. Therefore, our results were only partially consistent with our initial hypothesis because not all of the variables exhibited significant differences between the preand the post-test.

The coaches decided on a progressively decreasing TL during the last 3 weeks of the mesocycle. These changes, which consisted of a decrease in intensity and an increase in the number of tactical sessions, were consistent with the training methods documented in other studies that followed the same kind of a program to improve performance after a tapering period (Milanez et al., 2014). The TL assessment results during the mesocycle were consistent with the coaches' strategies. Both the methods of Edwards and RPEsession exhibited a progressive reduction in the TL over the last 3 weeks. Furthermore, there was a notable increase in monotony scores during the last three weeks. At the same time, the training strain did not decrease in the same proportion as the TLs represented by the RPEsession and Edwards indices. Previous studies have used monotony scores with psychological and physiological TL assessments (da Silva et al., 2015). It has been reported than high monotony scores are related to low TL variability, which may suggest the beginning of overtraining (Foster, 1998). When monotony and training strain were represented together with the weekly TL (Figure 1), we could observe that the sessions were not properly managed, because a significant reduction in TLs should result in a corresponding reduction in monotony and training strain, which was not the 
case. The increased monotony was due to low variety in training that could have been avoided by alternating intensity and volume between consecutive sessions (Comyns and Flanagan, 2013). The strong correlations found between the monotony and the specific stress and recovery indices of the RESTQ-sport can be attributed to the TL being more affected by psychological than physiological factors.

The performance test scores did not exhibit significant differences $(p>0.05)$ between the preand the post-test of the analyzed mesocycle. There was a little improvement in the results, which was far from what was expected after applying a tapering strategy during the last weeks of the season. These results are consistent with those of Oliveira et al. (2013), in which the changes in YoYo test performance are evident during the preseason, but not during the season, despite training.

Changes in the physiological performance test ( $\triangle$ Yo-YoIR1 and $\Delta$ Cooper) during the last mesocycle of the season only exhibited significant and large correlations with $\triangle$ RMSSD. This HRV variable did not exhibit any significant differences between the pre- and post-test period, and yielded similar results as the other performance variables. These findings are consistent with those of Flatt et al. (2017).

Meanwhile, the variables of the frequency domain of the HRV exhibited a significant decrease. This finding could be interpreted as showing that the team was coming to the end of the season and beginning to enter a phase of overtraining or that the players displayed relatively high indices of stress, aspects that were not reflected by the design of the loads or a decrease in players' performance. Other tennis (Thiel et al., 2011) and women's soccer (Flatt and Esco, 2015) studies that included an intense training period reported that lower HRV variables were not associated with decreased performance. Our results are similar to those of previous Yo-Yo IR1 test results, and the Yo-Yo IR1 improvement was not related to the relative changes in shortterm HRV. These changes could explain the absence of a correlation with the HRV variables (Buchheit et al., 2011; Oliveira et al., 2013). However, the HRV does not have to reflect the recovery of all systems since there are many different stressors unrelated to training (e.g., social, educational, occupational, economic, nutritional, travel, and time-related stress) (Flatt et al., 2017; Tian et al., 2013).

The use of the RESTQ-sport combined with physiological, non-invasive outcome measurements such as HRV has several precedents for monitoring the effects of the TL simultaneously with changes in stress and recovery (Dupuy et al., 2013; Morales et al., 2014; Thiel et al., 2011). Previous studies have reported similar results when comparing stress and physiological variables. For instance, Morales et al. ( 2014) reported decreased HRV values with higher levels of stress and lower recovery after 4 weeks of intense training with judo athletes; performance variables exhibited a significant decrease. Other studies have found that HRV and RESTQ-sport showed the same behavior, but did not correlate with athletes' performance (Dupuy et al., 2013; Thiel et al., 2011).

In the present study, the general stress and general recovery indices of the RESTQ-sport did not exhibit significant differences. Nevertheless, the sport-specific stress and sport-specific recovery scales demonstrated significant differences ( $\mathrm{p}<$ 0.05). These results might suggest overtraining, although here this conclusion has been ruled out due the results of the Cooper and Yo-Yo IR1 tests, which indicated stable athletes' performance. These findings suggest that motivational factors or training monotony likely resulted in the higher sport-specific stress scores and lower sport-specific recovery at the end of the season compared with the baseline status. In this sense, the results presented here are in agreement with those of Thorpe et al. (2015). This author affirmed that psychometric variables predicted fatigue in elite soccer players better than other markers such as HRV or Counter-Movement Jump.

The frequency-domain HRV results exhibited significant differences between the preand post-test, indicating decreased HRV and higher sympathetic ANS activation in response to the stressful situation (Stanley et al., 2013). According to Bosquet et al. (2008), HRV measured in the frequency domain is moderately reliable, but requires a highly standardized protocol to control for breathing, time of the day, temperature, luminosity, and noise, among other factors. Recent studies have considered timedomain variables, like the log RMSSD, to be the best indicator for athletic monitoring because this 
variable appears to be uninfluenced by the breathing rate and is more reliable than frequencydomain variables (Plews et al., 2014).

Our study meets the Task Forcestandardized protocol requirements to control for the influence of external factors (Task Force, 1996). Even so, our results are controversial in the HRV domain because the frequency domain exhibited significant differences between the pre- and the post-test and the time domain did not. The same situation occurred in the RESTQ-sport results; only the specific sport variables exhibited significant differences. A possible explanation for these results is that time-domain variables were more sensitive to external factors because of the increased anxiety produced by the overlap of the school exam period with the end of the training season and the associated decrease in motivation (D'Ascenzi et al., 2013).

The influence of the female menstrual cycle on HRV made it impossible to compare the groups more than once per month. Moreover, several previous studies have reported the effect of the female period on physiological functions such as HRV; sex-hormone fluctuations are basic physiological factors that continuously affects body functioning in women. Such studies have found increased LF components accompanied or unaccompanied by decreased HF components in HRV during the luteal phase compared with the follicular phase and indicated a predominant sympathetic activity in the luteal phase and a dominant vagal activity in the follicular phase (de Zambotti et al., 2013). The majority of previous studies based their results on analyses of frequency-domain and non-linear HRV variables, and it is rare to find studies that report the effect of the female period on the time domain of HRV variables. Recently, Brar et al. (2015) observed the effect of the female period on HRV, and these authors concluded that time-domain variables, particularly the lnRMSSD, did not always exhibit significant differences, in contrast with frequencydomain variables. As a result, Brar et al. (2015) were unable to easily detect period-induced changes.

There are a number of limitations of this study that should be considered. The most important one pertains to the HRV data collection, because the most recent studies (Flatt et al., 2017; Flatt and Esco, 2015) recommend monitoring HRV on a weekly basis by comparing the evolution of the coefficient of variation of a single variable such as $\operatorname{lnRMSSD}$. In our study, the pre- and post-tests were performed 6 weeks apart. Also, several HRV variables in the temporal and frequency domains were analyzed considering other methodological recommendations such as the menstrual cycle of females and breathing control for the treatment of the data. In this sense, there was a loss of information throughout the entire process, but it was compensated for by greater methodological rigor. At the same time, the follow-up throughout the process ensured that information associated with other load factors of training (e.g., monotony and training strain) could be obtained. Future research may choose to focus on combining weekly lnRMSSD tracking and the monotony and strain values derived from the RPEsession. Yet another limitation of this study was the lack of a control group to confirm the results and thus increase the quality of the research. However, this was a highstandard sample, and it is very difficult to gain access to the athletes and have them participating in time commitments beyond their normal training routine. In the end, this study opted for an observational design under which all subjects underwent the same measurements and the same training process.

In conclusion, the combined analysis of physiological and psychological factors related to training of a soccer team contributes much more valuable information than if these analyses are performed separately.

Monitoring HRV does not reflect changes in all physiological systems. Therefore, such monitoring needs to rely on other factors unrelated to training such as psychological stress (social stress, sleep, well-being, etc.).

Monotony is an index derived from the RPEsession that can be very useful for distributing sessions in a suitable way and thereby avoiding the situation in which TLs act as stressors.

The analyses of different HRV domains can reveal contradictory results in female athletes because the frequency domain is highly affected by the menstrual phase and cannot be assessed during the same week for all members of an allfemale team. To avoid these disturbances, it is preferable to analyze time-domain variables because they are more stable and are not altered by menstruation or by the breathing rate and 
stationary conditions. For this reason, this measurement is ideal for this type of study of female athletes. Moreover, the lnRMSSD is the time-domain variable that exhibited a stronger correlation with the RESTQ-sport and the Cooper and Yo-Yo IR1 tests.

\section{References}

Algrøy E, Hetlelid KJ, Seiler S, Pedersen JIS. Quantifying training intensity distribution in a group of norwegian professional soccer players. Int J Sports Physiol Perform, 2011; 6(1): 70-81

Bangsbo J, Iaia FM, Krustrup P. The Yo-Yo intermittent recovery test. Sport Med, 2008; 38(1): 37-51

Borresen J, Lambert MI. The Quantification of Training Load, Effect on Performance. Sport Med, 2009; 39(9): 779-795

Bosquet L, Merkari S, Arvisais D, Aubert AE. Is heart rate a convenient tool to monitor over-reaching? A systematic review of the literature. Br J Sports Med, 2008; 42(9): 709-714

Brar TK, Singh KD, Kumar A. Effect of Different Phases of Menstrual Cycle on Heart Rate Variability (HRV). J Clin diagnostic Res, 2015; 9(10): 1-4

Buchheit M, Allen A, Poon TK, Modonutti M, Gregson W, Di Salvo V. Integrating different tracking systems in football: multiple camera semi-automatic system, local position measurement and GPS technologies. J Sports Sci, 2014; 32(20): 1844-1857

Buchheit M, Voss SC, Nybo L, Mohr M, Racinais S. Physiological and performance adaptations to an in-season soccer camp in the heat: Associations with heart rate and heart rate variability. Scand J Med Sci Sports, 2011; 21(6): e477-e485

Comyns T, Flanagan EP. Applications of the session rating of perceived exertion system in professional rugby union. Strength Cond J, 2013; 35(6): 78-85

D'Ascenzi F, Alvino F, Natali BM, Cameli M, Palmitesta P, Boschetti G, Bonifazi M, Mondillo S. Precompetitive assessment of heart rate variability in elite female athletes during play offs. Clin Physiol Funct Imaging, 2014; 34 (3): 230-236

da Silva CC, Goldberg TBL, Soares-Caldeira LF, Oliveira R dos S, de Paula Ramos S, Nakamura FY. The Effects of 17 Weeks of Ballet Training on the Autonomic Modulation, Hormonal and General Biochemical Profile of Female Adolescents. J Hum Kinet, 2015; 47(1): 61-71

de Zambotti M, Nicholas CL, Colrain IM, et al. Influence of the menstrual cycle on nonlinear properties of heart rate variability in young women. Am J Physiol Circ Physiol, 2013; 38(11): 2618-2627

Dupont G, Akakpo K, Berthoin S. The effect of in-season, high-intensity interval training in soccer players. J Strength Cond Res, 2004; 18(3): 584-589

Dupuy O, Bherer L, Audiffren M, Bosquet L. Night and postexercise cardiac autonomic control in functional overreaching. Appl Physiol Nutr Metab, 2013; 38(2): 200-208

Edwards S. The Heart Hate Monitor Book. Sacramento: Fleet Feet Press; 1993

Flatt AA, Esco MR, Nakamura FY. Individual heart rate variability responses to preseason training in high level female soccer players. J Strength Cond Res, 2017; 31(2): 531-538

Flatt AA, Esco MR. Smartphone-derived Heart Rate Variability and Training Load in a Female Soccer Team. Int J Sports Physiol Perform, 2015; 10(8): 994-1000.

Foster C, Florhaug J a, Franklin J, Gottschall L, Hrovatin LA, Parker S, Doleshal P, Dodge C. A New Approach to Monitoring Exercise Training. J Strength Cond Res, 2001; 15(1): 109-115

Foster C, Hector LL, Welsh R, Schrager M, Green MA, Snyder AC. Effects of specific versus cross-training on running performance. Eur J Appl Physiol Occup Physiol, 1995; 70(4): 367-372

Foster C. Monitoring training in athletes with reference to overtraining syndrome. Med Sci Sports Exerc, 1998; 30(7): 1164-1168

Giles D, Draper N, Neil W. Validity of the Polar V800 heart rate monitor to measure RR intervals at rest. Eur J Appl Physiol, 2016; 116(3): 563-571

Gonzalez-Boto R, Salguero A, Tuero C, Marquez S. Concurrent validity of the Spanish version of the RecoveryStress Questionnaire for Athletes (RESTQ-Sport). Rev Psicol del Deport, 2009; 18(1): 53-72 
Kaikkonen P, Nummela A, Rusko H. Heart rate variability dynamics during early recovery after different endurance exercises. Eur J Appl Physiol, 2007; 102(1): 79-86

Kellmann M, Kallus KW. Recovery-stress questionnaire for athletes: user manual. Champaign, IL: Human Kinetics; 2001

Laux P, Krumm B, Diers M, Flor H. Recovery-stress balance and injury risk in professional football players: a prospective study. J Sports Sci, 2015; 33(20): 2140-2148

Malone JJ, Di Michele R, Morgans R, Burgess D, Morton JP, Drust B. Seasonal Training-Load Quantification in Elite English Premier League Soccer Players. Int J Sports Physiol Perform, 2015; 10(4): 489-497

McArdle WD, Katch FI, Katch VL. Exercise physiology: nutrition, energy, and human performance. Lippincott Williams \& Wilkins; 2010

Milanez VF, Ramos SP, Okuno NM, Boullosa DA, Nakamura FY. Evidence of a non-linear dose-response relationship between training load and stress markers in elite female futsal players. J Sport Sci Med, 2014; 13(1): 22-29

Morales J, Álamo JM, García-Massó X, Buscà B, López JL, Serra-Añó P, González LM. Use of heart rate variability in monitoring stress and recovery in judo athletes. J Strength Cond Res, 2014; 28(7): 1896-1905

Oliveira RS, Leicht AS, Bishop D, Barbero-Álvarez JC, Nakamura FY. Seasonal changes in physical performance and heart rate variability in high level futsal players. Int J Sports Med, 2013; 34(5): 424-430

Plews DJ, Laursen PB, Le Meur Y, Hausswirth C, Kilding AE, Buchheit M. Monitoring training with heart rate variability: how much compliance is needed for valid assessment. Int J Sport Physiol Perform, 2014; 9: 783-790

Stanley J, Peake JM, Buchheit M. Cardiac parasympathetic reactivation following exercise: implications for training prescription. Sport Med, 2013; 43(12):1259-1277

Task Force of the European Society of Cardiology and the North American Society of Pacing and Electrophysiology. Standards of measurement, physiological interpretation, and clinical use. Task Force of the European Society of Cardiology and the North American Society of Pacing and Electrophysiology. Circulation, 1996; 93: 1043-1065

Thiel C, Vogt L, Bürklein M, Rosenhagen A, Hübscher M, Banzer W. Functional Overreaching During Preparation Training of Elite Tennis Professionals. J Hum Kinet, 2011; 28(1): 79-89

Thorpe RT, Strudwick AJ, Buchheit M, Atkinson G, Drust B, Gregson W. Monitoring Fatigue during the InSeason Competitive Phase in Elite Soccer Players. Int J Sports Physiol Perform, 2015; 10(8): 958-964

Tian Y, He Z, Zhao J, Tao, DL, Xu KY, Earnest CP, Mc Naughton LR. Heart rate variability threshold values for early-warning nonfunctional overreaching in elite female wrestlers. J Strength Cond Res, 2013; 27(6): $1511-1519$

\section{Corresponding author:}

\section{Dr. Jose Morales,}

c/ Císter, 34, 08022 Barcelona, Spain.

Tel. +34 932533000 Fax: +34 932533031

E-mail: josema@blanquerna.url.edu 